\title{
NONLINEAR SOLITON INTERACTION WITH ACOUSTIC SIGNALS: FOCUSING EFFECTS
}

\author{
O.C. Rodríguez ${ }^{1}$, S. Jesus ${ }^{1}$, Y. Stephan ${ }^{2}$, X. Demoulin ${ }^{2}$, M. Porter ${ }^{3}$ and E. Coelho ${ }^{4}$ \\ ${ }^{1}$ UCEH-Universidade do Algarve, Campus de Gambelas, 8000, Faro, Portugal \\ ${ }^{2}$ EPSHOM/CMO, BP426, F-29275, Brest, France \\ ${ }^{3}$ Science Applications International Corp., San Diego, USA. \\ ${ }^{4}$ Instituto Hidrográfico, 1296, Lisboa, Portugal
}

\begin{abstract}
The problem of nonlinear interaction of solitary wave packets with acoustic signals has been intensively studied in recent years. A key goal is to explain the observed transmission loss of shallow-water propagating signals, which has been found to be strongly time-dependent, anisotropic, and sometimes exhibited unexpected attenuation vs. frequency. Much of the existing literature considers the problem of signal attenuation in a static environment, without considering additional effects arising from groups of solitons evolving both in range and time. Hydrographic and acoustic data from the INTIMATE'96 experiment clearly exhibit the effects of soliton packets. However, in contrast with reported observations of signal attenuation, the observed transmission loss shows a pronounced signal enhancement that behaves like a focusing effect. This focusing is correlated with peaks in current, temperature, and surface tide. That correlation suggests that the nonlinear interaction of solitary wave packets with acoustic signals can lead to a focusing of the signal. To clarify this issue, hydrographic data was used to generate physically consistent distributions of "soliton-like" fields of temperature and sound velocity. These distributions were then used as input for a range-dependent normal-mode model. The results strongly support the hypothesis that the soliton field causes the observed signal enhancement.
\end{abstract}

\section{INTRODUCTION}

Naturally generated solitons can often be observed in coastal zones as a result of nonlinear interaction of the surface tide with the continental shelf. Acoustic propagation through such Solitary Wave Packets (SWPs) has been intensively studied in recent years (see for instance [1] and [2]). These SWPs have often been identified as the main cause of abnormal signal attenuations. Most of the studies analysed the problem of acoustic propagation through SWPs from a "static" point of view, since they did not consider the effects of SWPs that evolve in both range and time. A detailed analysis of current, thermistor, and acoustic data from the INTIMATE'96 experiment [3] reveals SWPs propagating across the experimental site. An interesting feature of the acoustic data, is an increase of signal amplitude which is clearly correlated with peaks in current, temperature, and surface tide. This increase of amplitude can be seen as a sort of focusing effect. To determine if this effect could be the result of acoustic propagation through a "dynamic" SWP, the hydrographic data from the experiment was used to develop physi- 
cally consistent distributions of range-dependent "soliton-like" fields of temperature and sound speed. These were then given as input to an acoustic propagation model. The simulation results strongly support the assumption that a SWP was responsible for the observed signal focusing.

This paper is organized as follows: section 2 presents a brief theoretical background of soliton propagation; this background simplifies the analysis of hydrographical data from the INTIMATE'96 experiment, which is described in section 3. The acoustic data, correlated with hydrographic data from the previous section, is discussed in section 4 and it is followed by corresponding acoustic simulations. The main conclusions of the paper are presented in section 5 .

\section{THEORETICAL BACKGROUND}

The starting point for the analysis of soliton propagation in a rotationless environment with complex stratification is the so-called Korteweg-de Vries equation (hereafter simply $\mathrm{KdV}$ ) for modal vertical displacement $\eta_{m}[4],[5]$ :

$$
\frac{\partial \eta_{m}}{\partial t}+C_{m} \frac{\partial \eta_{m}}{\partial x}+\alpha_{m} \eta_{m} \frac{\partial \eta_{m}}{\partial x}+\beta_{m} \frac{\partial^{3} \eta_{m}}{\partial x^{3}}=0
$$

where

$$
\begin{gathered}
\alpha_{m}=\frac{3}{2} s \frac{C_{m}}{D} \quad, \quad \beta_{m}=\frac{1}{2} d D^{2} C_{m}, \\
s=D \frac{\left\langle\phi_{m}^{3}\right\rangle}{\left\langle\phi_{m}^{2}\right\rangle} \quad, \quad d=\frac{1}{D^{2}} \frac{\left\langle\Psi_{m}^{2}\right\rangle}{\left\langle\phi_{m}^{2}\right\rangle}, \\
\frac{d^{2} \Psi_{m}}{d z^{2}}+\frac{N^{2}}{C_{m}^{2}} \Psi_{m}=0, \phi_{m}=\frac{d \Psi_{m}}{d z}, \Psi_{m}(0)=\Psi(D)=0,\left\langle\phi_{m} \phi_{n}\right\rangle=\left\langle N^{2} \Psi_{m} \Psi_{n}\right\rangle=0,
\end{gathered}
$$

$N$ is the buoyancy frequency, $D$ is the water depth, $x$ corresponds to the axis of propagation, \langle\rangle is an "inner product" defined as

$$
\langle\ldots\rangle=\int_{0}^{D} \ldots d z
$$

and $\Psi_{m}$ and $\phi_{m}$ are the Hydrostatic Normal Modes (hereafter HNMs) of the corresponding linear rotationless form of the hydrodynamic equations. In contrast with the corresponding equation for a homogeneous fluid [6], which admits a single soliton generation, it follows from Eq. (1) that by combining both nonlinear and stratification effects

it is possible to obtain an entire set of "modal" solitons with characteristics that depend on HNMs. Whether or not this explains the observations of propagating SWPs depends on the properties of the HNMs.

\subsection{The "Sech" solution}

For displacements having large enough amplitudes and steepness, the KdV equation admits the well-known soliton solution [7]

$$
\eta_{m}=\eta_{m}^{0} \operatorname{sech}^{2}\left(\frac{x-\mathcal{C}_{m} t}{\Delta_{m}}\right)
$$


where $\eta_{m}^{0}$ represents the peak amplitude of the modal soliton, which has a nonlinear characteristic width

$$
\Delta_{m}=\sqrt{\frac{12 \beta_{m}}{\alpha_{m} \eta_{m}^{0}}}
$$

and propagates with a nonlinear phase speed given by

$$
\mathcal{C}_{m}=C_{m}+\frac{\alpha_{m} \eta_{m}^{0}}{3}
$$

As seen from the above equations $\Delta_{m}$ is inversely proportional to the amplitude of the modal soliton, whereas $\mathcal{C}_{m}$ is linearly proportional to $\eta_{m}^{0}$; the implication is that the larger $\eta_{m}^{0}$, the faster the soliton propagates and the narrower or steeper the soliton is.

\subsection{The "Dnoidal" Solution}

Another solution to the $\mathrm{KdV}$ equation is [7]

$$
\eta_{m}=\eta_{m}^{0}\left[2 \operatorname{dn}_{(m, \mathrm{~S})}^{2}\left(\frac{x-\mathcal{C}_{m} t}{\Delta_{m}}\right)-\left(1-\mathrm{S}^{2}\right)\right]
$$

where the index $S$ is a function of the normalized variable $\tau=x / C_{m} t$ and $\operatorname{dn}_{S}(\varphi)$ is the "dnoidal" Jacobi elliptic function. The shapes of the "dnoidal" solution agree well with the backscattered profiles measured from SAR images ([5],[8]). As $\mathrm{S} \rightarrow 1$ the above expression becomes the "sech" profile. From a dynamical point of view there are significant differences between this and the previous type of solutions: Eq. (6) describes a SWP with a single modal soliton, which propagates in time and space without deforming its shape (see Fig. 1, on top). In contrast, Eq. (9) describes a perturbation with an evolving profile (see Fig. 1, on bottom), resembling the evolution of soliton packets. The number of solitons within a packet depends on the values of $\tau$ and S. The importance of this point will be discussed in section 4 when performing the acoustic simulations.

\subsection{Temperature perturbations}

It follows from hydrodynamic coupled equations for horizontal currents and displacement [4] that the horizontal components of fluid velocity depend linearly on vertical soliton displacement. Nevertheless, from a tomographic point of view, the system of hydrodynamic equations does not provide a physical basis for expanding the temperature field, and thus the sound-speed field. To address the tomographic issue, let us recall the thermodynamic equation [9]

$$
\frac{D}{D t}\left(\rho C_{v} T\right)=\nabla\left(k_{T} \nabla T\right)+Q_{T},
$$

where $C_{v}$ denotes the specific heat at constant volume, $k_{T}$ is the thermal conductivity, and $Q_{T}$ represents all sources and sinks of heat. Linearizing and solving this differential equation (see the appendix) one obtains:

$$
T \approx T_{0}(z)+\frac{d T_{0}}{d z} \sum_{m} T_{m} \eta_{m} \Psi_{m}
$$

which shows that the vertical structure of temperature is related to the HNM $\Psi_{m}$ (in contrast with horizontal currents, which depend only on $\phi_{m}$ ). The horizontal dependence remains linearly related to the vertical displacement and this approximation becomes linear when $d T_{0} / d z \approx$ constant. 
The theoretical description of soliton propagation will be used in the following section to understand the current and temperature features found in hydrographic records from the INTIMATE'96 experiment.

\section{EXPERIMENTAL DATA}

The INTIMATE'96 experiment ([3],[10]) performed during June 1996, North of Lisbon (see Fig. 2, on top) involved the collaboration of the following institutions: the Universidade do Algarve (UALG), Faro, the Instituto Hidrográfico (IH), Lisbon (both from Portugal), and the Centre Militaire Oceanographique (SHOM), Brest, France. The project team received also support from the Saclant Undersea Research Centre (SACLANTCEN), La Spezia, Italy, which lended the Vertical Linear Array (hereafter VLA). The experiment was conceived with the main goal of applying ocean acoustic tomography to the detection and inversion of the internal tide. The French oceanographic vessel BO'DENTRECASTEAUX towed an acoustic source at $90 \mathrm{~m}$ depth, which emitted linear frequency modulated chirps sweeping from $300-800 \mathrm{~Hz}$ with a 2 -second duration. The transmissions were repeated every 8 seconds, then received on the 4-hydrophone VLA and telemetered back to the Portuguese vessel NRP ANDROMEDA. The hydrophones were located at 35, 70, 105 and $115 \mathrm{~m}$ depth. Signal transmissions were performed from north and west positions (see Fig. 2, on bottom), along range-independent and range-dependent acoustic tracks, respectively, with corresponding distances of approximately 5.6 and $6.4 \mathrm{kms}$. The bottom compressional speed and attenuation were estimated from coring measurements as 1750 $\mathrm{m} / \mathrm{s}$ and $0.9 \mathrm{~dB} /$ wavelength, respectively. During the experiment an intensive survey of thermistor, CTD, XBT and ADCP data was performed near the position of the VLA and at the source location. This allowed for the calculation of Empirical Orthogonal Functions (hereafter EOFs, see Fig. 3) and HNMs (see Fig. 4), and a high degree of correlation was found between both sets of functions [10].

In particular, the mean sound-speed profile exhibits a typical summer shallow-water profile which decreases with depth (see Fig. 5), the corresponding values of the discretized profile are shown in Table 1. The smooth downward refracting gradient of the profile contrasts significantly with usual schematic two-layer representations.

A preliminary step in the analysis of current and temperature data consisted in calculating the dependence of $\Delta_{m}$ on $\eta_{m}^{0}$ for some of the HNMs (see Eq. (7)). Those calculations revealed that not every pair $\left(\Psi_{m}, \phi_{m}\right)$ is "allowed" to generate modal solitons. For $m>1$, the characteristic soliton width $\Delta_{m}$ becomes complex, and this implies that the characteristics of the SWPs depend only on $\Psi_{1}$ and $\phi_{1}$.

To detect the propagation of SWPs across the experimental site (and on the basis of the previous result) one can take advantage of the correlation between EOFs and HNMs, and calculate the empirical "modal" amplitudes of hydrographic data for the first EOF. Those amplitudes were separated with a Butterworth filter into low-pass and high-pass components with the cutoff frequency chosen in order to obtain a "tidal" band (with periods longer than 4h) and a "buoyancy" band (with shorter periods). Due to the low sampling frequency $(\sim 1 / 10$ minutes $)$ the estimation of vertical displacement from thermistor data did not resolve the structure of SWPs. Nevertheless, it was expected to "capture" some of the solitons within a packet -if present- in the high-pass component. This data processing did not provide any physical information about the direction and phase velocity of SWPs since the measurements were taken at a single location. The results of filtering are shown in Fig. 6. In all cases -and particularly in the bottom plots of temperature records- it can be seen the presence of significant "peaks" (the position 
of the first three peaks is denoted with arrows), which are not distributed randomly, but appear to be repeated at each tidal cycle. Those peaks are located slightly behind the maxima of the corresponding tidal component, which is remarked by the vertical dash lines starting at each peak's position and crossing the low-pass component of modal amplitude. The position of the second peak is "missing" in the high-pass modal amplitude of $u$, which can be due to the low temporal resolution of hydrographic data. However, its expected position is shown to enhance the general "alignment" between the soliton peaks and the corresponding maxima of tidal components. Peaks in the current data reach amplitudes up to $40 \mathrm{~cm} / \mathrm{s}$, which is close to the corresponding maximal value reported by Apel et al. $[5]$.

\section{ACOUSTIC SIMULATIONS AND COMPARISON WITH REAL DATA}

Based on the analysis of temperature records the first step consisted on searching for acoustic perturbations of the received signal, temporally correlated with any of the peaks of the high-pass component of temperature. To accomplish this task part of the acoustic transmissions from the north source position to the VLA was processed, during a time interval of $\Delta t=3 \mathrm{~h}$ around the third temperature peak (near $166.75 \mathrm{~d}$, see Fig. 6, on bottom). The processing consisted in calculating the following quantity:

$$
-20 \log _{10}|\hat{p}(f)|
$$

where $\hat{p}(f)$ corresponds to the monochromatic component at frequency $f$, of the Fourier transform of the received signal $p(t)$. The quantity defined by Eq. (12) will be called -formally - as "relative transmission loss" (hereafter RTL). The processed acoustic data revealed a sharp pattern of acoustic perturbations (near $166.765 \mathrm{~d}$, see Fig. 7, on top). The pattern is complex but it reveals an increase in signal amplitude across a wide band of frequencies. As remarked earlier, this can be seen as a sort of focusing effect. At a single frequency the perturbation pattern appears to be poorly resolved, due to the superposition of noise on each frequency component. Therefore, some averaging was applied to different RTL curves over a temporal window of 200 seconds. The curves were further smoothed in time with a low-pass filter. The RTL curve at $430 \mathrm{~Hz}$ (see Fig. 7, on bottom), shows the focusing effect more clearly. The overall amplitude excursion of the RTL corresponds approximately to $9 \mathrm{~dB}$.

Besides the propagation of soliton packets across the experimental site it seems unlikely to find other physical mechanisms that can account for the RTL perturbation described above. In particular, for the INTIMATE'96 environment, the propagation of the surface tide does not affect the depth of the thermocline. Therefore, the tide can not lead to significant interactions of the acoustic signal with the bottom.

Unfortunately, the temporal correlation between the temperature peak commented previously and the acoustic data is not evident. This might be due to the significant difference between the sampling rates of temperature records, one sample every 10 minutes, and acoustic transmissions, with one emission every 8 seconds. To clarify this issue one can exploit the theoretical knowledge on soliton propagation and generate "soliton-like" fields of temperature and sound velocity, which can be used as input for an acoustic propagation model. The primary goal of the simulations is to obtain a qualitative agreement between the modeled and the measured dependences of RTL along time.

An important theoretical question of soliton propagation is the choice of initial conditions that define the starting shape $\eta_{m}(x, 0)$ and starting amplitude $\eta_{m}^{0}$ of the SWP. A 
complete discussion of this problem should handle the analysis of the forcing mechanisms of soliton generation, which is an issue beyond the scope of this paper. For this reason, and also because SWPs in our environment depend only on $\Psi_{1}$ and $\phi_{1}, \eta_{1}^{0}$ was estimated from direct measurements, through the analysis of isotherms from thermistor data. To accomplish this task the mean depth of each isotherm was calculated, and the corresponding isotherm oscillations along time were corrected to the mean depth. This gave a distribution of corrected isotherms, from which it was selected the one that exhibited the narrowest peak of amplitude, which was considered to be representative of the initial soliton amplitude. That gave $\eta_{1}^{0} \approx 25 \mathrm{~m}$. Furthermore, from Eqs. (2),(3),(4) and (7) one can predict that $C_{1}=44 \mathrm{~cm} / \mathrm{s}, \alpha_{1}=0.012 \mathrm{~s}^{-1}$ and $\beta_{1}=300.4 \mathrm{~m}^{3} / \mathrm{s}$, while from Eq. (8) one predicts that $\mathcal{C}_{1}=54 \mathrm{~cm} / \mathrm{s}$. This value of soliton speed is close to the measured values discussed in [5].

The "solitonized" fields of temperature are calculated from Eq. (11). Furthermore, each temperature profile is converted to sound-speed using the well-known Mackenzie's formula. Those transformations reflect the fact that the propagation of the SWP across the acoustic waveguide leads to time-dependent -and range-dependent- perturbations of the sound-speed profiles. Those perturbations on sound-speed affect dynamically the acoustic signal, mainly due to the dependence of refraction and surface/bottom interactions, on time and range.

The expected geometry of soliton propagation is shown in Fig. 8, where $\theta$ represents the direction of propagation. Since the thermistor chains were located slightly to the east of the VLA, and because the temperature perturbation occurs before the acoustic perturbation, one can assume that the SWP "starts" propagating south-east from the VLA. According to the previous figure the effective width of the soliton front is $\Delta_{e}=\Delta / \sin \theta$, where $\Delta$ is the width of the front along the direction of propagation ${ }^{1}$. Moreover, the effective velocity of soliton propagation is $V_{e}=\mathcal{C}_{1} \sin \theta$. In this way, one starts calculating the transmission loss (hereafter TL) using the mean sound-speed profile, "displaces" the SWP a distance $V_{e} \Delta t$ (with $\Delta t=2$ minutes) towards the north position of the acoustic source, and calculates the TL again. The displacement is repeated until the SWP covers a temporal window of 3 hours.

A preliminary set of simulations was performed with the acoustic model C-SNAP [11], by calculating the TL at $f=430 \mathrm{~Hz}$, for a "sech" profile, and for a set of four "dnoidal" SWPs. Each "dnoidal" soliton packet contained an integer number of solitons, up to four. Those packets were calculated by modifying, through trial and error, the parameters $\mathrm{S}$ and $\tau$. The angle of propagation was considered as $\theta=15^{\circ}$, which corresponds to the estimated direction of propagation of the internal tide [10]. The shape of the "dnoidal" packets did not change over time, which is an assumption based on observations ${ }^{2}$. The results showed a complex dependence of TL on time, where one can observe high levels of attenuation, but also levels of signal focussing. The result that resembles better the curve of RTL was obtained for the case of a "dnoidal" SWP with four solitons (see Fig. 9). The modeled dependence of TL on time exhibits the pattern of attenuation, focusing and attenuation again, as the SWP approaches and passes over the VLA. Further simulations with other values of $\theta$, using both the "sech" and "dnoidal" soliton profiles, revealed an oscillating-like highly nonlinear dependence of TL on $\theta$. These simulations gave further consistency to the soliton hypothesis, since the acoustic model predicted certain degrees of signal focusing at particular positions of the soliton packets. Nevertheless, none of the

\footnotetext{
${ }^{1}$ Do not confuse this width with the characteristic soliton width $\Delta_{m}$.

${ }^{2}$ For instance, the soliton packet described in [12], and observed at a few tens of miles from the INTIMATE'96 experimental site, propagated shoreward keeping a stable shape during almost $12 \mathrm{~h}$.
} 
simulations reproduced a focusing effect in qualitative agreement with the one observed.

\section{CONCLUSIONS}

On the basis of this analysis the following conclusions can be drawn: 1)nonlinear approximations to hydrodynamic equations for a rotationless environment admit the generation of "modal" solitons with characteristics that depend on HNMs; 2)the "sech" and "dnoidal" modal solutions of the KdV equation correspond to different dynamics of soliton propagation: the "sech" profile describes a single soliton, which propagates in time and space without changing its shape, while "dnoidal" profiles evolve both in time and space and for certain parameter choices can give a better description of SWPs; 3)current and temperature hydrodynamic fields depend linearly on vertical modal displacements, which can be obtained as solutions of the KdV equation; such solutions can be used to generate physically consistent fields of temperature and sound velocity; 4)separation of current and thermistor records of the INTIMATE'96 experiment into low-pass and high-pass components shows evidence of soliton groups propagating across the experimental site; 5)one of these groups is coincident with a strong perturbation of the acoustic signal which, when analysed in detail, reveals an increase of signal amplitude, i.e., an effect similar to signal focusing and 6)simulations of acoustic propagation through "soliton-like" fields of sound velocity show a similar effect of signal focusing and confirm the assumption that a SWP may be responsible for the observed acoustic perturbation. 


\section{REFERENCES}

[1] Zhou J., Zhang X., and Rogers P. Resonant interaction of sound wave with internal solitons in the coastal zone. J. Acoust. Soc. America, 90(4):2042-2054, October 1991.

[2] Caille G.W. et al. Overview of the joint chine-u.s. yellow sea-96 experiment. In Zhang R. abd Zhou J., editor, Shallow-Water Acoustics, pp. 17-22, Beijing-China, April 1997. China Ocean Press. proceedings of the SWAC'97 Conference.

[3] Démoulin X., Stéphan Y., Jesus S., Coelho E., and Porter M. "Intimate'96: A shallow water tomography experiment devoted to the study of internal tides". In Proceedings of $S W A C^{\prime} 97$, pp. 485-490, Beijing, April 1997. China Ocean Press. Edited by Zhang $\mathrm{R}$. and Zhou J.

[4] Ostrovsky L.A. Nonlinear internal waves in a rotating ocean. Oceanology, Vol. 18 No.2, pp. 119-125, 1978.

[5] Apel J.R. et al. An overview of the 1995 swarm shallow-water internal wave acoustic scattering experiment. IEEE Journal of Oceanic Engineeering, 22(3):465-500, July 1997.

[6] Gabov S. A. Introduction to the theory of non-linear waves. Ed. by the Moscow State University, Moscow, 1988. (in russian).

[7] Apel J., Orr. M., Finette S., and Lynch J. A new model for internal solitons and tides on the continental shelf. In Zhang R. and Zhou J., editors, Shallow-Water Acoustics, pp. 219-225, Beijing, April 1997. China Ocean Press. Proceedings of the SWAC'97 Conference.

[8] Apel J. et al. The new jersey shelf shallow water acoustic random medium propagation experiment (swarm). In Zhang R. and Zhou J., editors, Shallow-Water Acoustics, pp. 213-218, Beijing, April 1997. China Ocean Press. Proceedings of the SWAC'97 Conference.

[9] LeBlond P.H. and Mysak L.A. Waves in the Ocean. Elsevier Scientific Publishing Company, New York, 1989.

[10] Rodríguez O.C., Jesus S., Stephan Y., Démoulin X., Porter M., and Coelho E. Internal tide acoustic tomography: Reliability of the normal modes expansion as a possible basis for solving the inverse problem. In Alippi A. and Cannelli G.B., editors, Proc. of the 4th. European Conference on Underwater Acoustics, pp. 587-592, Rome, Italy, 21-25 September 1998.

[11] Ferla C.M., Porter M.B., and Jensen F.B. C-SNAP: Coupled SACLANTCEN normal mode propagation loss model. SACLANT UNDERSEA RESEARCH CENTRE (SM274), La Spezia, Italy, 1993.

[12] D.R.G. Jeans, and Sherwin T.J. Solitary internal waves on the Iberian shelf. MORENA scientific and technical report no.44, University of Wales, UK, 1996. 


\section{List of Figures}

Fig.

1 Spatial and temporal evolution of soliton modal displacement $\eta_{1}$, for a "sech" profile [top], and for a "dnoidal" profile [bottom]. Soliton parameters are $\eta_{1}^{0}=5 \mathrm{~m}, \Delta_{1}=196 \mathrm{~m}$ and $\mathcal{C}_{1}=54.2 \mathrm{~cm} / \mathrm{s}$ (values taken from

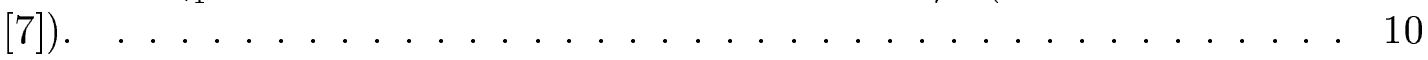

2 Chosen area of the INTIMATE'96 experiment [top] and bathymetry of the experimental site [bottom] (contour depths in $\mathrm{m}$ ). . . . . . . . . . 11

3 EOFs for current components $u$ (continuous line) and $v$ (dot-dash line) [top] and for temperature $T$ [bottom]. . . . . . . . . . . . 12

4 Normalized HNMs $\Psi_{m}$ (continuous line) and $\phi_{m}=d \Psi_{m} / d z$ (dot-dash line). 12

5 Mean velocity profile from CTD-IH. . . . . . . . . . . . 13

6 Modal amplitudes of horizontal current components $u$ [top], $v$ [middle] and temperature $T$ [bottom]; velocity is in $\mathrm{cm} / \mathrm{s}$ and temperature in Celsius degrees. The second peak is missing in the modal amplitude of $u$ but its expected position is shown to enhance the general comparison. . . . . . 14

7 Relative transmission loss over frequency [top] and averaged and smoothed relative transmission loss at $430 \mathrm{~Hz}$ [bottom]. . . . . . . . . . 15

8 Considered geometry of soliton propagation across the experimental site. - 16

9 Simulation result for a "dnoidal" SWP with 4 solitons. . . . . . . . . 16 

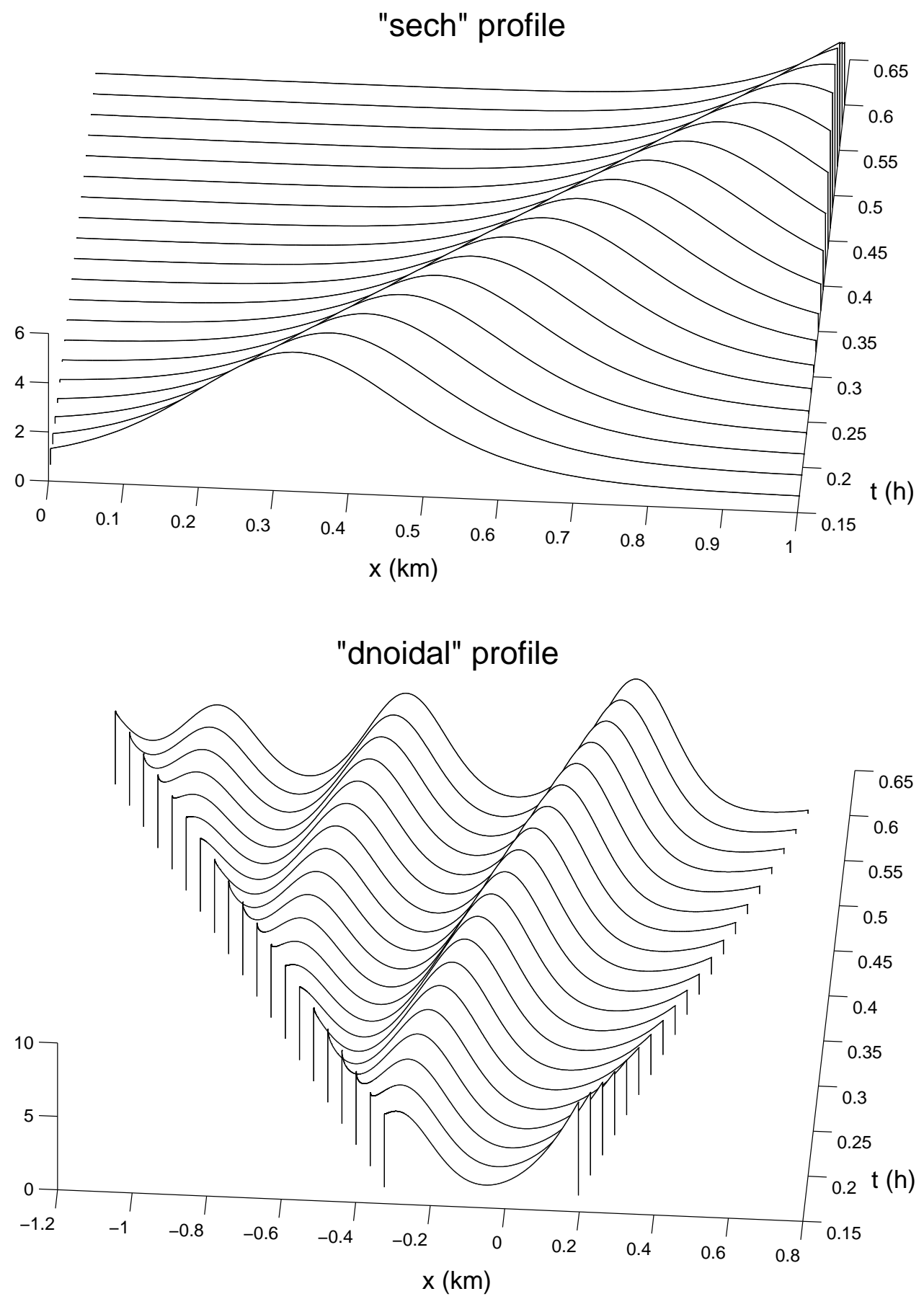

Figure 1: Spatial and temporal evolution of soliton modal displacement $\eta_{1}$, for a "sech" profile [top], and for a "dnoidal" profile [bottom]. Soliton parameters are $\eta_{1}^{0}=5 \mathrm{~m}, \Delta_{1}$ $=196 \mathrm{~m}$ and $\mathcal{C}_{1}=54.2 \mathrm{~cm} / \mathrm{s}$ (values taken from [7]). 

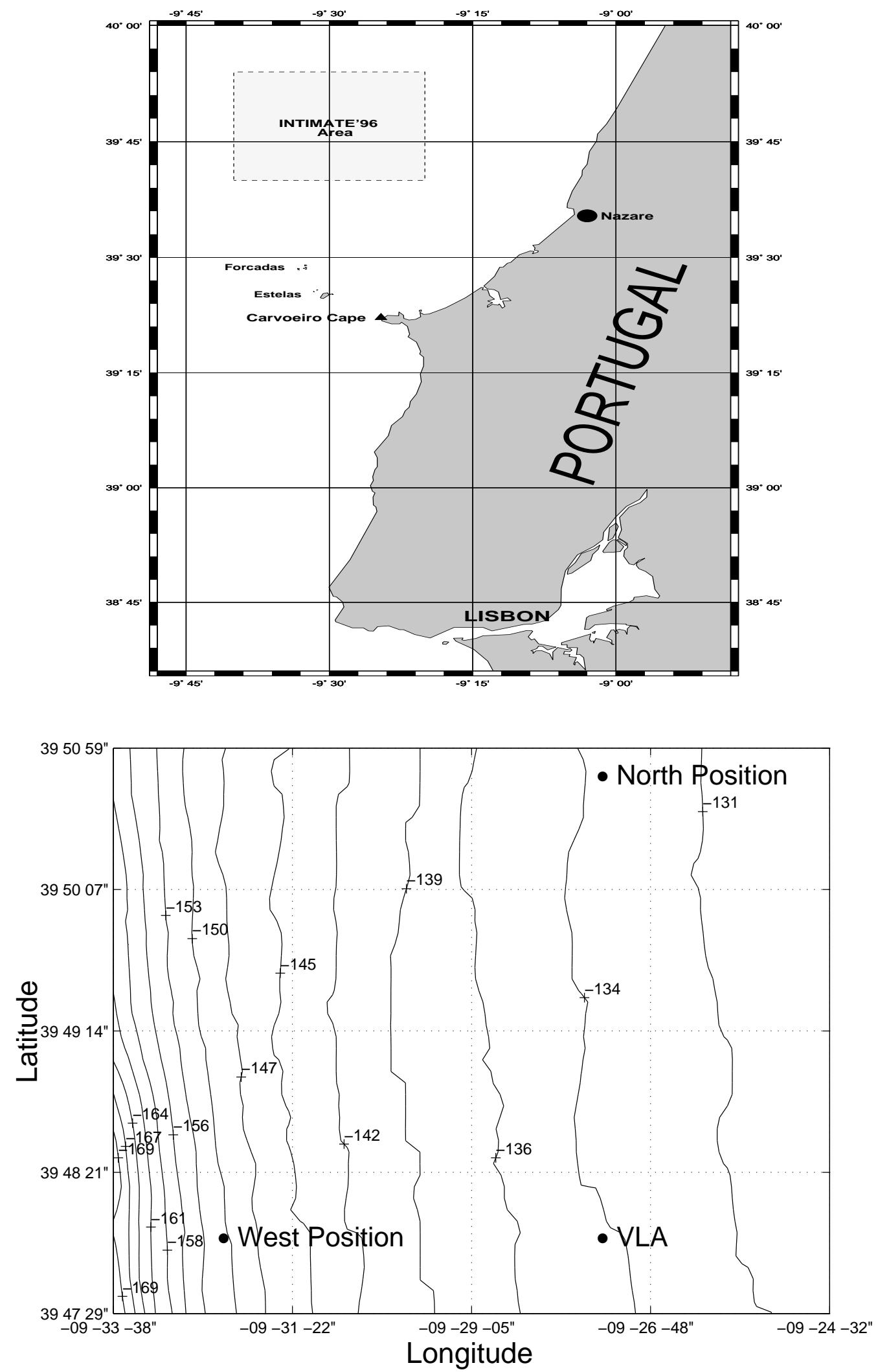

Figure 2: Chosen area of the INTIMATE'96 experiment [top] and bathymetry of the experimental site [bottom] (contour depths in $\mathrm{m}$ ). 

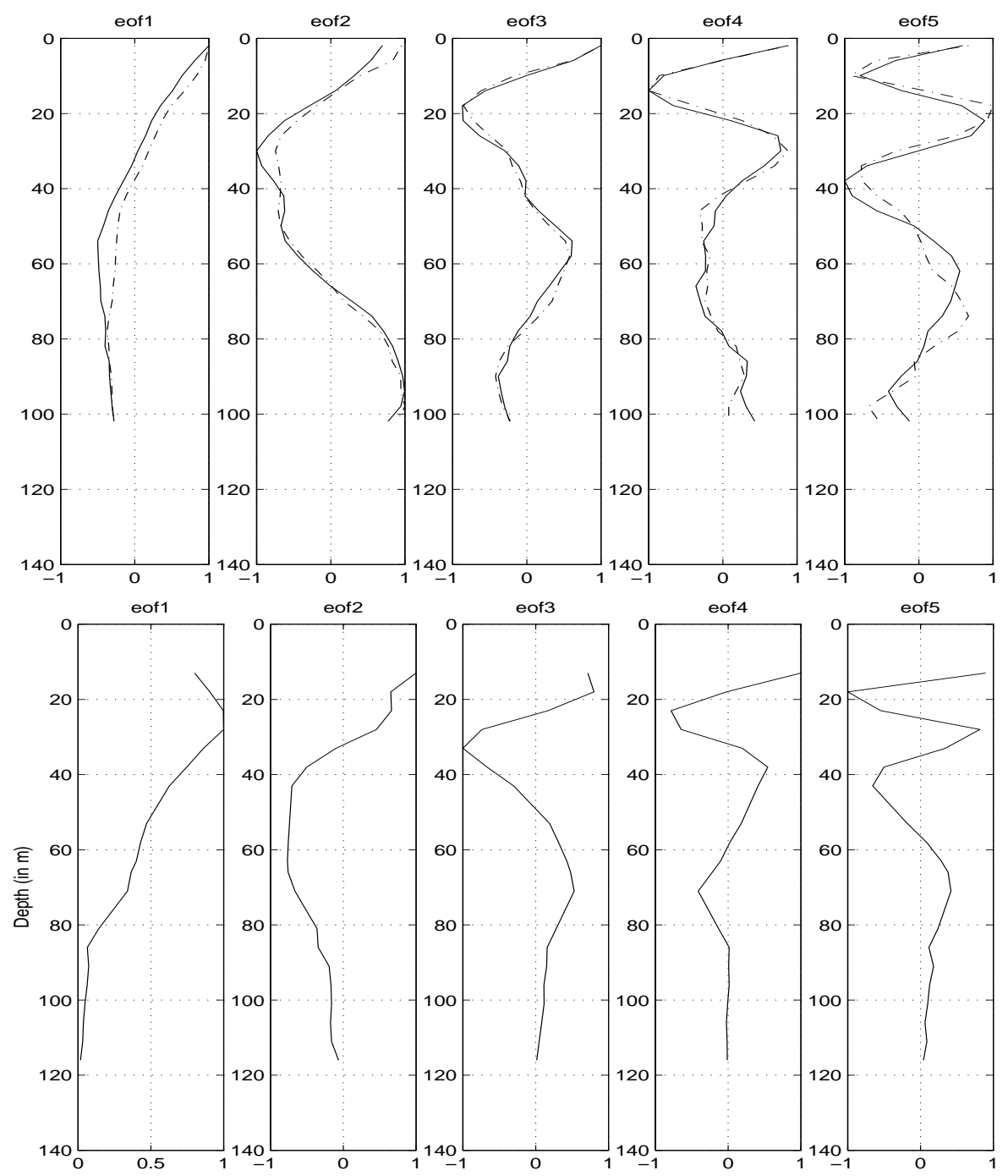

Figure 3: EOFs for current components $u$ (continuous line) and $v$ (dot-dash line) [top] and for temperature $T$ [bottom].

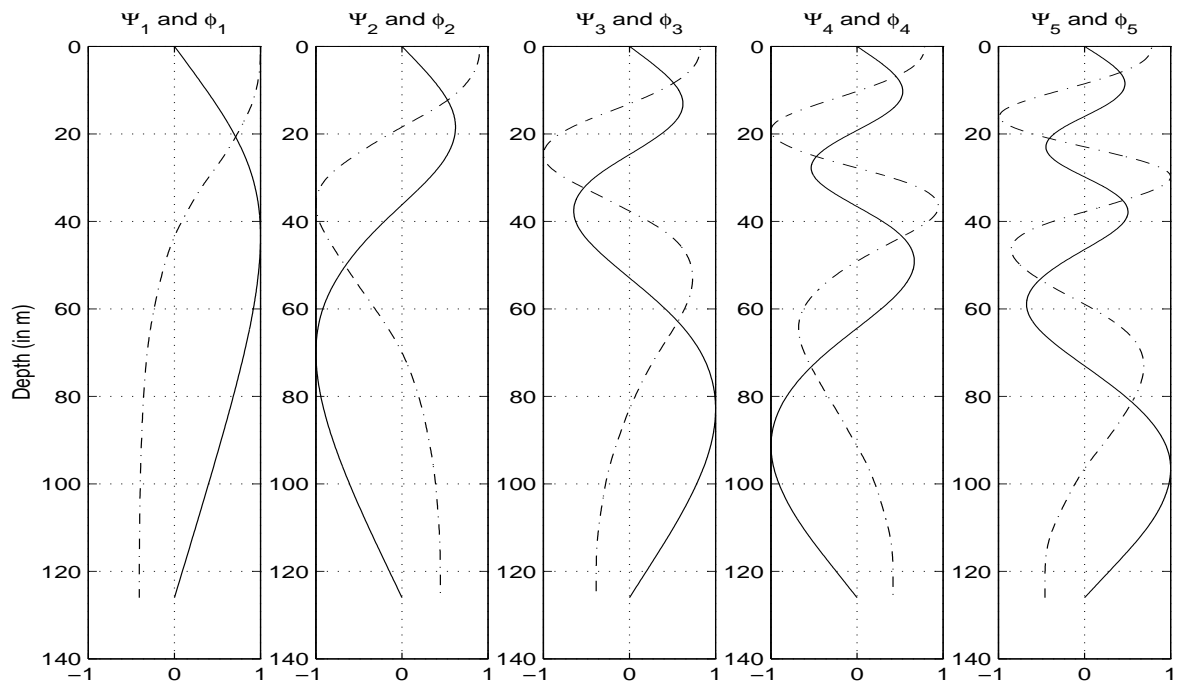

Figure 4: Normalized HNMs $\Psi_{m}$ (continuous line) and $\phi_{m}=d \Psi_{m} / d z$ (dot-dash line). 


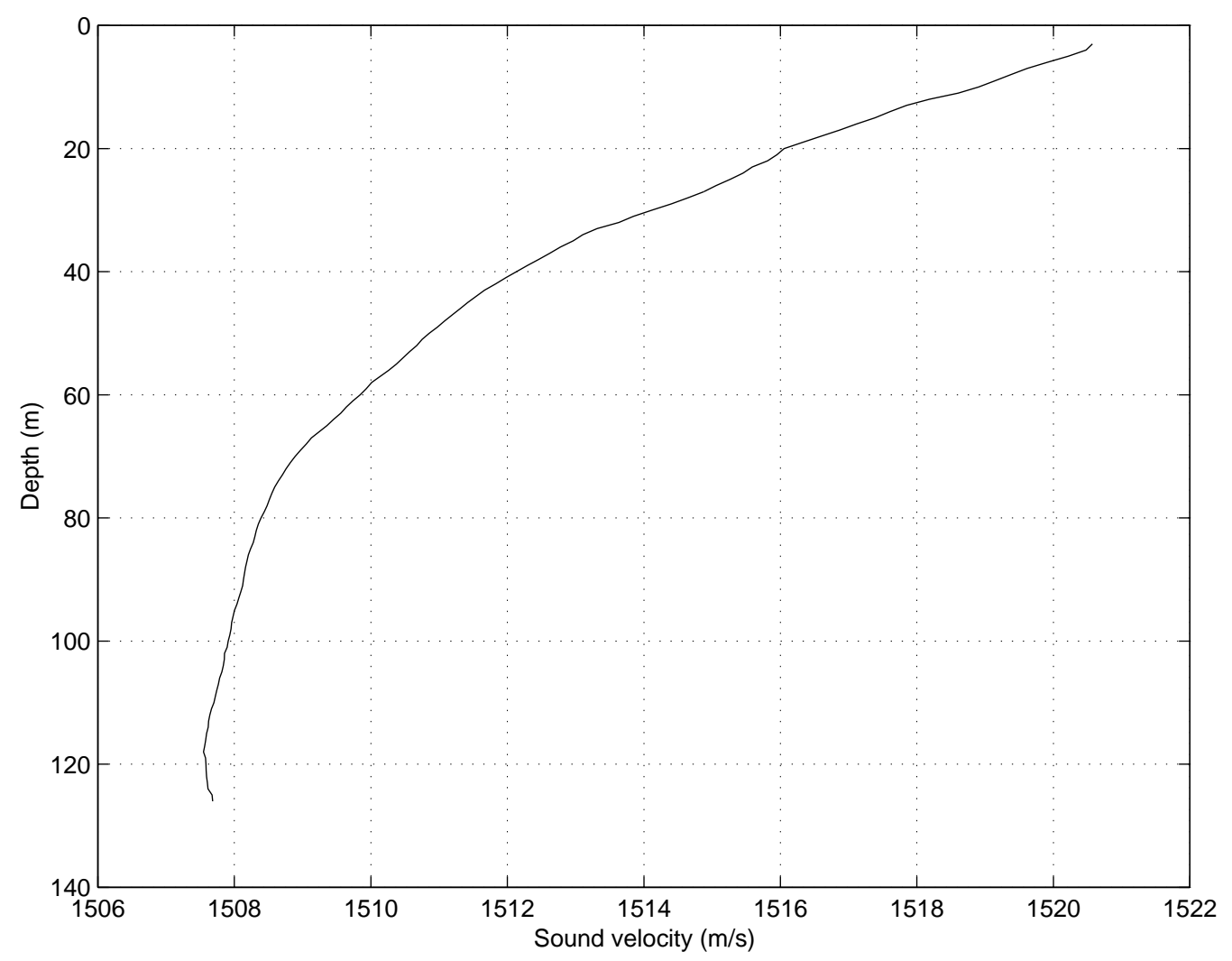

Figure 5: Mean velocity profile from CTD-IH.

\begin{tabular}{||c|c|c|c||}
\hline \hline Depth $(\mathrm{m})$ & Sound Velocity $(\mathrm{m} / \mathrm{s})$ & Depth $(\mathrm{m})$ & Sound Velocity $(\mathrm{m} / \mathrm{s})$ \\
\hline \hline 3 & 1520.6 & 65 & 1509.4 \\
\hline 5 & 1520.2 & 70 & 1508.9 \\
\hline 10 & 1518.9 & 75 & 1508.6 \\
\hline 15 & 1517.4 & 80 & 1508.4 \\
\hline 20 & 1516.1 & 85 & 1508.2 \\
\hline 25 & 1515.3 & 90 & 1508.1 \\
\hline 30 & 1514.1 & 95 & 1508.0 \\
\hline 35 & 1512.9 & 100 & 1507.9 \\
\hline 40 & 1512.1 & 105 & 1507.8 \\
\hline 45 & 1511.4 & 110 & 1507.7 \\
\hline 50 & 1510.9 & 115 & 1507.6 \\
\hline 55 & 1510.4 & 120 & 1507.6 \\
\hline 60 & 1509.8 & 125 & 1507.7 \\
\hline \hline
\end{tabular}

Table 1: Discretized values of the mean sound-speed profile. 

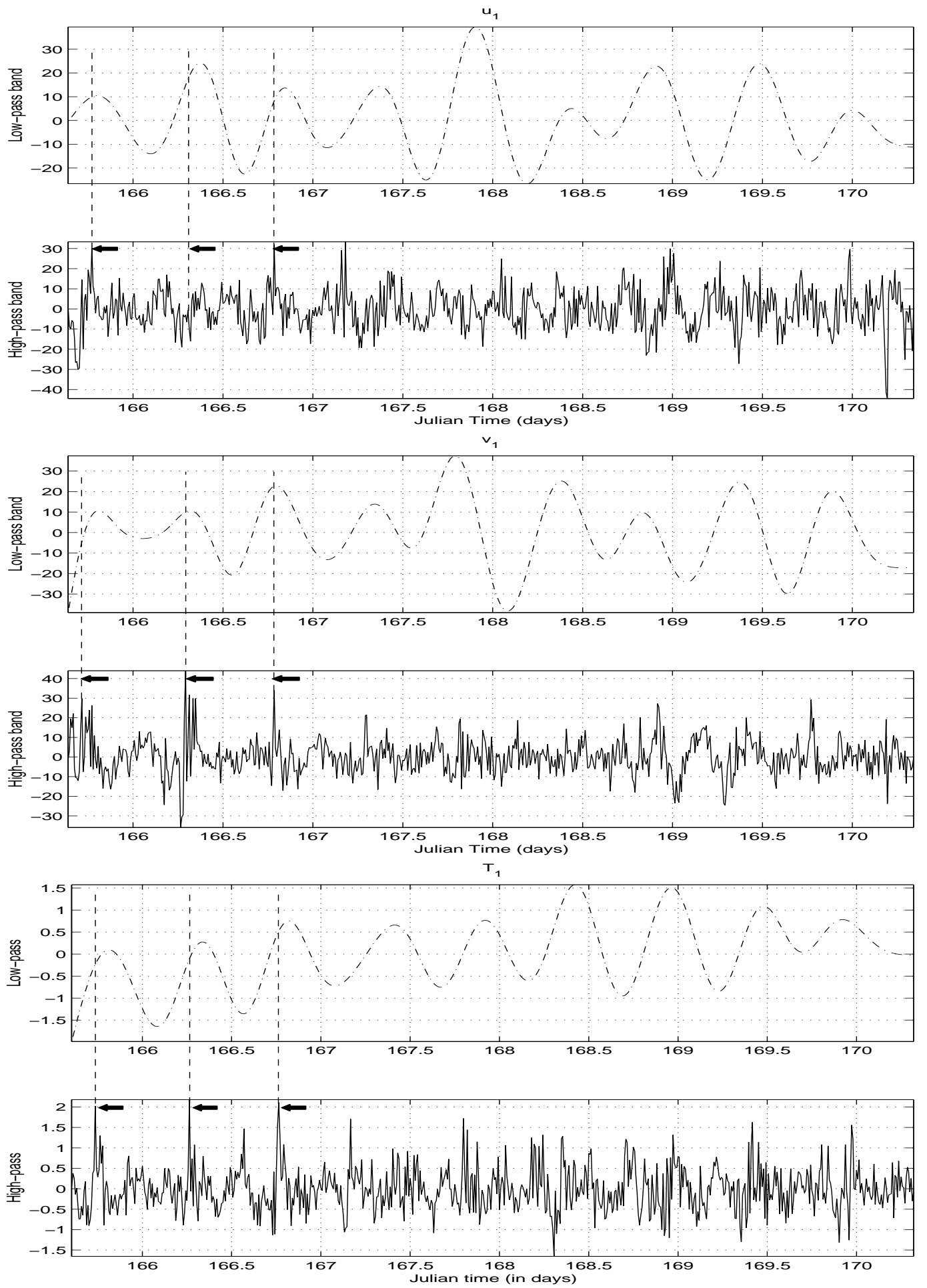

Figure 6: Modal amplitudes of horizontal current components $u$ [top], $v$ [middle] and temperature $T$ [bottom]; velocity is in $\mathrm{cm} / \mathrm{s}$ and temperature in Celsius degrees. The second peak is missing in the modal amplitude of $u$ but its expected position is shown to enhance the general comparison. 

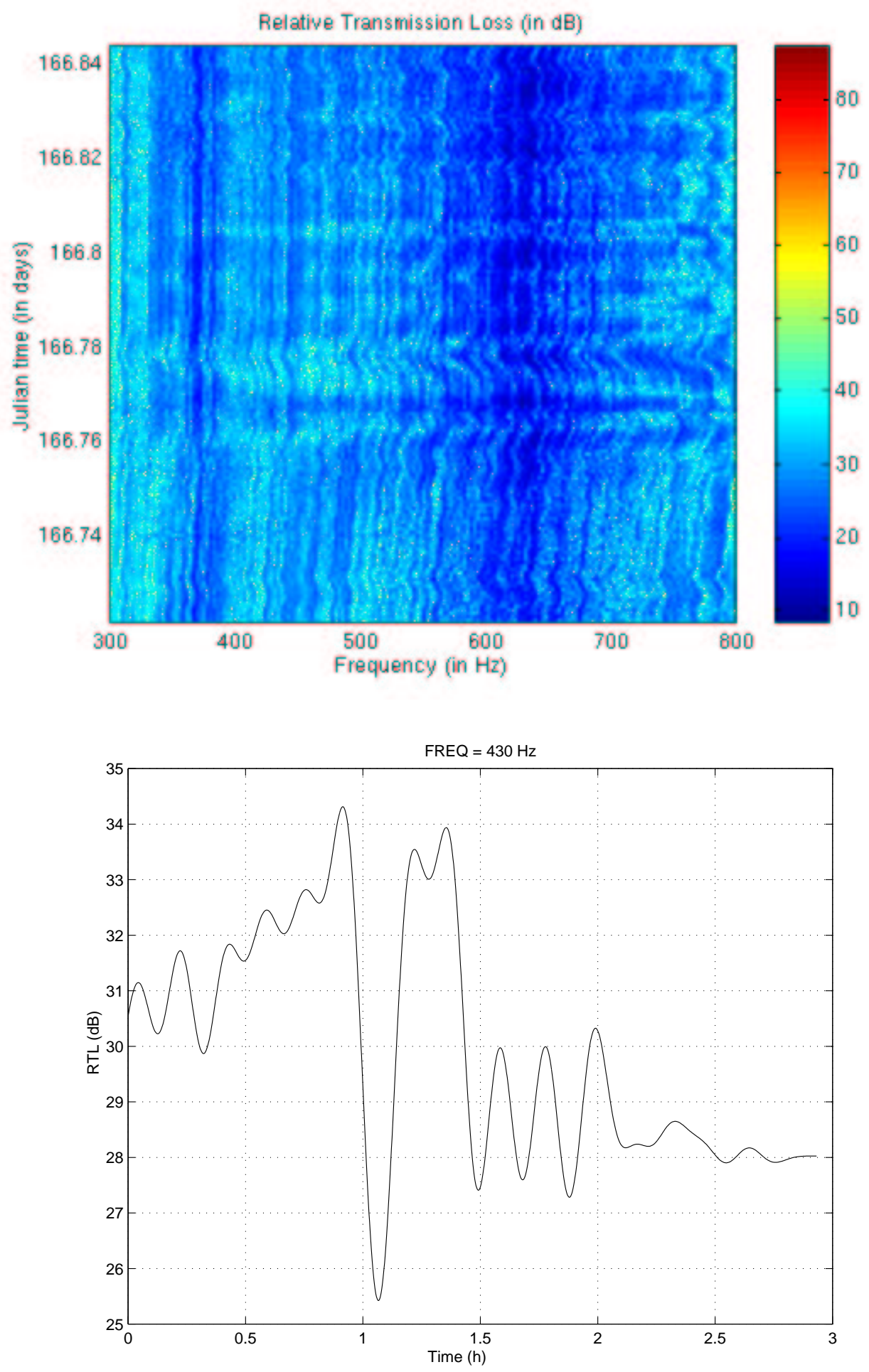

Figure 7: Relative transmission loss over frequency [top] and averaged and smoothed relative transmission loss at $430 \mathrm{~Hz}$ [bottom]. 


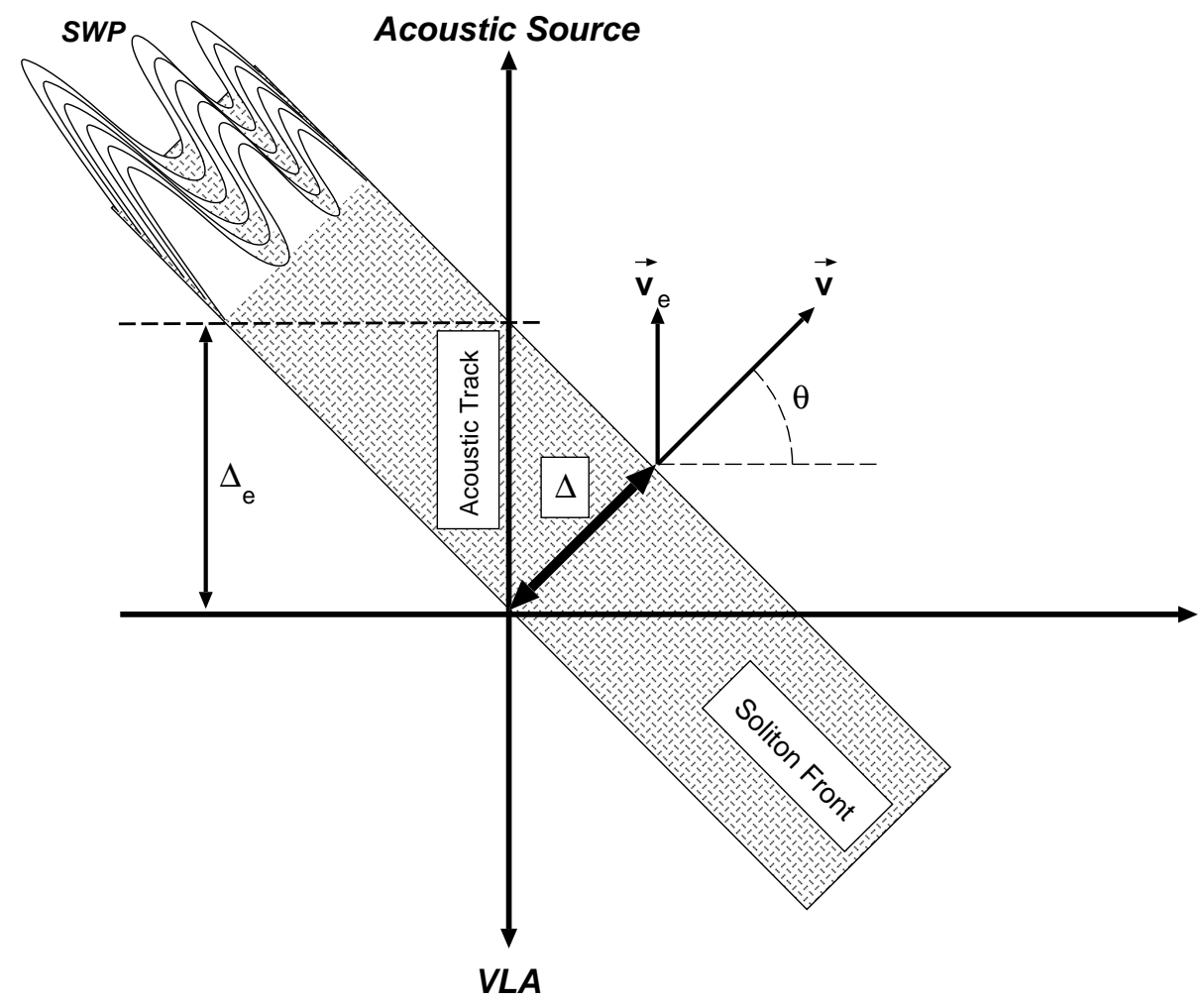

Figure 8: Considered geometry of soliton propagation across the experimental site.

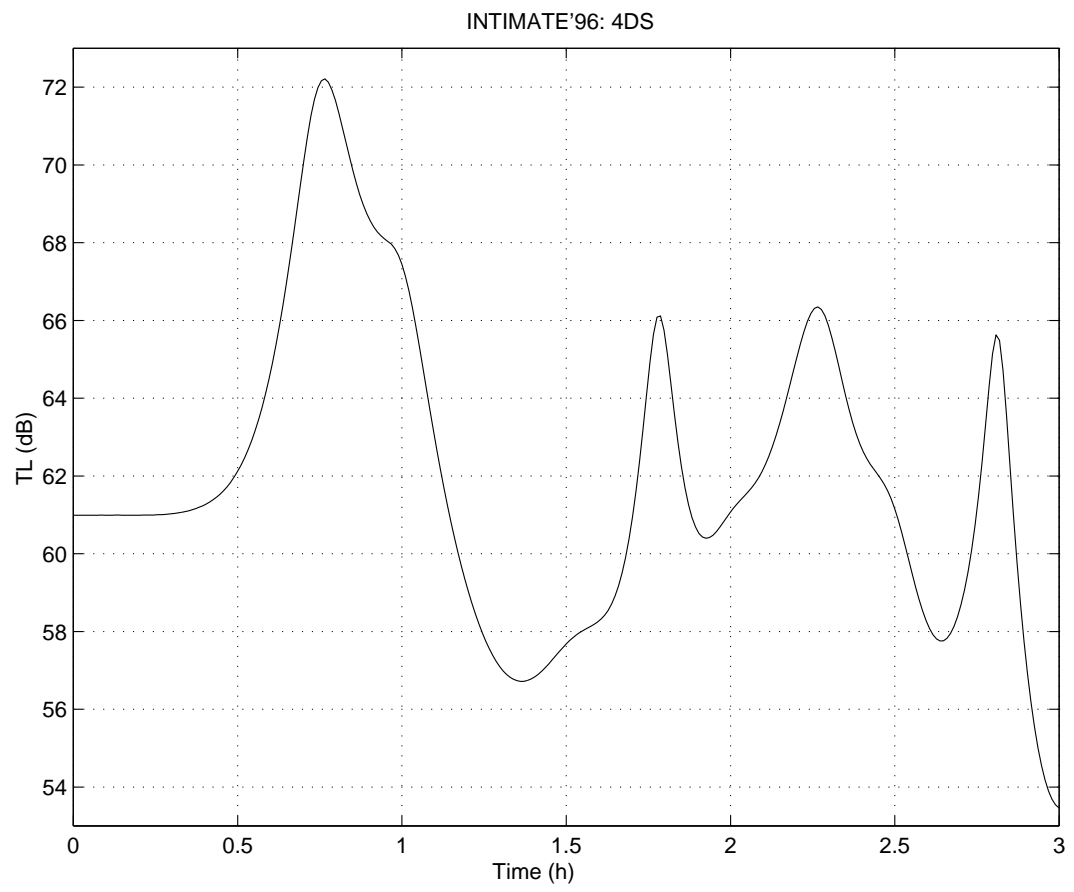

Figure 9: Simulation result for a "dnoidal" SWP with 4 solitons. 


\section{A Appendix}

It can be shown [5] that the dynamic fields of currents $(u, v, w)$ can be expanded in terms of HNMs $\Psi_{m}$ and $\phi_{m}=d \Psi_{m} / d z$ as follows:

$$
(u, v)=\sum_{m}\left(u_{m}, v_{m}\right) \eta_{m} \phi_{m}, \text { and } w=\sum_{m} w_{m} \Psi_{m} \frac{\partial \eta_{m}}{\partial t}
$$

where $\eta_{m}$ represents modal displacement, and $\left(u_{m}, v_{m}, w_{m}\right)$ are coefficients of modal amplitude for the current components $u, v$ and $w$. For $\eta_{m}$ being calculated in meters, and $t$ in seconds, $w_{m}$ will be a dimensionless parameter while $u_{m}$ and $v_{m}$ will have dimensions $\mathrm{m} / \mathrm{s}$. By taking $k_{T}=0$ and constant $\left(c_{v}, \rho\right)$ Eq. (10) becomes:

$$
\frac{\partial T}{\partial t}+u \frac{\partial T}{\partial x}+v \frac{\partial T}{\partial y}+w \frac{\partial T}{\partial z}=0
$$

In the general case it is not clear which terms can be neglected and which ones can not. However, by neglecting coupling mechanisms and taking into account that an important feature of soliton propagation is the significant dynamics of the perturbation along the depth axis, one can neglect the second and third non-linear terms, and rewrite Eq. (A.2) as follows:

$$
\frac{\partial T}{\partial t}+w \frac{\partial T}{\partial z}=0
$$

Now, recalling the general structure of expansions [Eqs. (A.1)] let us consider that

$$
T(x, y, z, t) \approx T_{0}(z)+T^{\prime}, \text { and } T^{\prime}=\sum_{m} T_{m} \Psi_{m} \gamma_{m}
$$

In the last expression $\gamma_{m}$ is an unknown function, while $T_{m}$ corresponds to a dimensionless coefficient of modal amplitude for temperature. Both $\gamma_{m}$ and $T_{m}$ should be chosen in order to ensure the consistency of Eq. (A.3). Using Eqs. (A.4) it can be obtained that:

$$
\frac{\partial T}{\partial t}=\sum_{m} T_{m} \Psi_{m} \frac{\partial \gamma_{m}}{\partial t}
$$

Furthermore, neglecting again coupling mechanisms between modes, and through further linearization, the second term in Eq. (A.3) can be approximated as:

$$
w \frac{\partial T}{\partial z} \approx \frac{d T_{0}}{d z} \sum_{m} w_{m} \Psi_{m} \frac{\partial \eta_{m}}{\partial t}
$$

Substituting Eq. (A.5) and Eq. (A.6) into Eq. (A.3) it follows automatically that

$$
T_{m}=-w_{m} \text { and } \gamma_{m}=\eta_{m} \frac{d T_{0}}{d z}
$$

The minus sign indicates that the time oscillations of $w$ and $T$ have a phase difference of $\pi$ radians. The last pair of equations lead to Eq. (11). 